\title{
Patrones de prescripción de medicamentos para la diabetes mellitus tipo 2 en cinco departamentos de Colombia, en 2014
}

\author{
Patterns of Medication Prescriptions for Diabetes Mellitus Type 2 in \\ five Departments of Colombia in 2014
}

\section{Padrões de prescrição de medicamento para a Diabetes Mellitus tipo 2 em cinco departamentos da Colômbia em 2014}

\author{
Noël C. Barengo ${ }^{1}$, Sandra Camacho², Pilar Andrea López³, Paul Anthony Camacho4, Ángel Alberto García ${ }^{5}$, Jaime Alejandro \\ Hincapié6, Alexander Maceneth ${ }^{7}$, Patricio López-Jaramillo8 \\ 1 Organización para la Excelencia de la Salud, Bogotá, Colombia. Herbert Wertheim College of Medicine, Florida \\ International University, Estados Unidos. nbarengo@fiu.edu \\ 2 Organización para la Excelencia de la Salud, Bogotá, Colombia \\ 3 Organización para la Excelencia de la Salud, Bogotá, Colombia \\ 4 Fundación Oftalmológica de Santander, Bucaramanga, Colombia \\ 5 Hospital Universitario San Ignacio, Pontificia Universidad Javeriana, Bogotá, Colombia \\ 6 Clínica Integral de Diabetes, Medellín, Colombia \\ 7 Centro Médico Oftalmológico, Ibagué, Colombia \\ 8 Fundación Oftalmológica de Santander, Bucaramanga, Colombia
}

Recibido: 24/04/2017. Aprobado: 29/05/2018. Publicado: 30/04/2018

Barengo NC, Camacho S, López PA, Camacho PA, García ÁA, Hincapié JA, Maceneth A, López-Jaramillo P. Patrones de prescripción de medicamentos para la diabetes mellitus tipo 2 en cinco departamentos de Colombia, en 2014. Rev. Fac. Nac. Salud Pública. 2018;36(2):58-65. Dor:10.17533/udea.rfnsp.v36n2a08

\section{Resumen}

Objetivo: Describir los patrones de prescripción de los medicamentos para la diabetes mellitus tipo 2 (DM2) y comorbilidades de pacientes atendidos en cinco instituciones prestadoras de servicios de salud de Colombia. Metodología: Estudio descriptivo transversal, en el cual se revisaron las historias clínicas de 5098 pacientes con DM2, atendidos en centros de atención ambulatoria ubicados en cinco ciudades colombianas entre el $1 .^{\circ}$ de enero y el 31 de diciembre de 2014. Cada uno de los pacientes con DM2 tenía al menos dos consultas ambulatorias registradas durante el periodo de estudio. La recolección de la información se hizo mediante una encuesta electrónica. Para la categorización de los medicamentos se usaron las guías 
nacionales e internacionales para el tratamiento de la diabetes. $\mathrm{El}$ análisis de los datos fue realizado utilizando el programa IBM SPSS $^{\circledR}$ Statistics versión 21. Resultados: El medicamento de más frecuente prescripción fueron las biguanidas (59\%) y las sulfonilureas (28\%). La prescripción de inhibidores de la dipeptidil peptidasa-4 fue $7 \%$ y la frecuencia de prescripción de agonista del receptor del péptido similar al glucagón tipo 1 (AR GLP-1) fue de $2 \%$. El medicamento con mayor frecuencia de prescripción como monoterapia fueron las biguanidas $(22 \%)$. La combinación más frecuente fue biguanida y las sulfonilureas (21\%), seguida de biguanida e insulina (10\%), y otras combinaciones. El $27 \%$ pacientes con DM2 no recibió ningún tratamiento farmacológico para la diabetes. Con respecto a los medicamentos para comorbilidades, el $52 \%$ de los pacientes utiliza al menos un tipo de antihipertensivo, el $39 \%$ usa al menos un tipo de hipolipemiante y el $35 \%$ utiliza ácido acetilsalicílico. Conclusiones: Las biguanidas fueron el medicamento con mayor frecuencia de prescripción, seguido de las sulfonilureas. Uno de cada cuatro pacientes no tenía registro de prescripción de medicamentos. El uso de ácido acetilsalicílico como prevención del riesgo cardiovascular fue menor al esperado.

---------Palabras clave: atención primaria de salud, diabetes mellitus tipo 2, prescripciones de medicamentos, Colombia.

\section{Abstract}

Objective: to describe the patterns of medication prescriptions for diabetes mellitus type 2 (DM2) and the comorbidity of patients treated in five health care institutions in Colombia. Methodology: descriptive cross-sectional study carried out checking the medical records of 5098 patients with DM2 treated at the outpatient service centers in five Colombian cities between January 1 and December 31 of 2014. Each patient with DM2 had a record of at least two outpatient appointments registered during the time of this study. The information was collected through electronic surveys. National and international guidelines on diabetes treatment were used to categorize the medications. The SPSS $^{\circledR} 21$ software was used to analyze the data. Results: the most frequently prescribed medications were biguanides (59\%) and sulfonylureas (28\%). The prescription of inhibitors for Dipeptidyl peptidase- 4 was $7 \%$ and the frequency of prescription of glucagon-like peptide-1 receptor agonists (AR
GLP-1) was $2 \%$. The medication with the highest frequency of prescription as monotherapy were biguanides $(22 \%)$. The most frequent combination was biguanide and sulfonylureas (21\%). The second most frequent combination was biguanide with insulin (10\%), and other combinations. $27 \%$ of patients with DM2 did not receive any pharmacological treatment for diabetes. Regarding the medicines for comorbidity, $52 \%$ of patients use at least one type of antihypertensive drug, 39\% use at least one type of hypolipidemic drug and 35\% uses acetylsalicylic acid. Conclusions: biguanides were the most frequently prescribed medication, sulfonylureas came after. One in four patients did not have a record of medicine prescription. The prescription of acetylsalicylic acid to prevent cardiovascular risk was lower than expected.

-------Key words: primary health care, diabetes mellitus type 2, medicine prescription, Colombia

\section{Resumo}

Objetivo: descrever os padrões de prescrição dos medicamentos para a Diabetes Mellitus tipo 2 (DM2) e de comorbilidades de pacientes atendidos em cinco instituições de serviço de saúde da Colômbia. Metodologia: estudo descritivo transversal, no qual revisaram-se as histórias clínicas de 5098 pacientes com DM2, atendidos em centros de atendimento ambulatorial localizados em cinco cidades colombianas, entre 1 de janeiro e 31 de dezembro de 2014. Cada paciente com DM2 tinha pelo menos duas consultas ambulatoriais registradas durante o período do estudo. A informação coletou-se através de inquérito eletrônico. Para a categorização dos medicamentos, utilizaram-se os guias nacionais e internacionais para o tratamento da diabete. A análise dos dados realizou-se utilizando o programa SPSS ${ }^{\circledR} 21$. Resultados: os medicamentos de prescrição mais frequente foram biguanidas (59\%) e as sulfoniluréias (28\%). A prescrição de inibidores da dipeptidil peptidase IV foi $7 \%$ e a frequência de prescrição de agonista do receptor do péptido si- milar ao glucagão tipo 1 (AR GLP-1) foi de 2\%. O medicamento com mais frequência de prescrição como monoterapia foi as biguanidas (22\%). A combinação mais frequente foi biguanida e sulfoniluréias (21\%). A segunda combinação mais frequente foi biguanida com insulina (10\%), e outras combinações. $27 \%$ dos pacientes com DM2 não recebeu tratamento farmacológico nenhum para a diabetes. Respeito dos medicamentos para comorbilidades, $52 \%$ dos pacientes utiliza pelo menos um tipo de anti-hipertensivo, 39\% utiliza pelo menos um tipo de hipolipemiante e 35\% utiliza ácido acetilsalicílico. Conclusões: as biguanidas foram o medicamento com mais frequência de prescrição, e depois as sulfoniluréias. Um de quatro pacientes não rinha registro de prescrição de medicamentos. $\mathrm{O}$ uso de ácido acetilsalicílico como prevenção do risco cardiovascular foi menor do que se esperava.

-Palavras-chave: atendimento primário de saúde, Diabetes Mellitus tipo 2, prescrições de medicamentos, Colômbia. 


\section{Introducción}

A nivel mundial, el número de personas con diabetes mellitus (DM) crece rápidamente. Para el 2014 existían 415 millones de personas diagnosticadas con DM en todo el mundo [1]. Este número se cree que aumentará hasta 642 millones en el año 2040.

La prevalencia de diabetes mellitus tipo 2 (DM2) en Colombia varía entre el 4 y el $8 \%$, dependiente del rango de edad de la población de investigación. Según un estudio del 2010, la prevalencia de la DM2 en las zonas rurales fue menor del $2 \%$ [2].

El tratamiento de la DM2 comprende la implementación de conductas saludables, así como las intervenciones farmacológicas.

Desde el punto de vista de la salud pública, es importante conocer el perfil de la prescripción y de la distribución efectiva de medicamentos para los pacientes con DM2, para analizar y mejorar la calidad de su atención [3-5]. Después de la entrada en vigencia de la Ley 100 de 1993, se observó que más de la mitad de los pacientes con DM no recibía medicamentos antidiabéticos incluidos en el Plan Obligatorio de Salud (Pos), que hace parte del Sistema General de Seguridad Social en Salud (sGsss) [6].

En Colombia, los patrones de prescripción farmacológica indicados a los pacientes con DM han sido descritos por algunos investigadores durante la pasada década [3-5]. Sin embargo, no existen estudios recientes sobre dichos patrones.

Desde 1993, el sGsss colombiano ha sufrido modificaciones sustanciales - ajustes en el plan de beneficios-y se han introducido nuevos medicamentos en el país. Ello permitió que, durante los últimos 20 años, en la medida en que se han logrado desarrollar nuevas tecnologías y tratamientos costo-efectivos, dichos medicamentos se pusieran progresivamente a disposición de los médicos, para el manejo de los pacientes diabéticos.

Estudios sobre los patrones de prescripción farmacológica para pacientes con DM son importantes para generar directrices sobre el uso racional de medicamentos en el contexto de la salud pública, para apoyar la toma de decisiones clínicas, para el desarrollo de políticas y directrices, y para asignar eficientemente los recursos.

De esta manera, el objetivo de este estudio fue determinar los patrones de prescripción farmacológica para pacientes con DM2 en Colombia, en el escenario de atención ambulatoria, y describir los medicamentos empleados para comorbilidades entre el $1 .^{\circ}$ de enero y el 31 de diciembre de 2014.

\section{Metodología}

\section{Material}

Se realizó un estudio descriptivo transversal sobre la prescripción de medicamentos. Se llevó a cabo una revisión de historias clínicas de una cohorte de pacientes atendidos durante el año 2014 en el servicio de consulta externa dirigida a pacientes con DM2, en cinco instituciones prestadoras de servicios de salud (IPS) en las ciudades de Barranquilla, Bucaramanga, Ibagué, Medellín y Montería.

Se usó un muestreo no probabilístico, donde las instituciones fueron seleccionadas por su conveniente accesibilidad y proximidad para el grupo de investigación, a través de contactos personales. Solo instituciones de centros urbanos fueron invitadas, y también aquellas que contaran con un programa estructurado para el manejo de la diabetes. Se invitó solo a centros que tenían un volumen de 1000 atenciones en el año 2013. Además, se intentó que las instituciones participantes fueran de áreas geográficas diferentes.

Se asumieron como elegibles los registros de pacientes con diagnóstico de alta de la DM (CIE-10: E100E149) y que fueron atendidos en las instituciones entre enero y diciembre de 2014. El análisis se concentró en pacientes que tuvieron al menos dos citas médicas durante el año calendario. Pacientes embarazadas y pacientes con DM tipo 1 fueron excluidos de este estudio.

\section{Recolección de información sobre patrón de pres- cripción farmacológica}

La información fue recolectada mediante una herramienta electrónica, para generar y alimentar la base de datos al momento mismo de la recolección. El equipo de trabajo que participó en la recolección de la información en cada una de las instituciones estaba formado por personas del área de la salud (médicos, jefes de enfermería, auxiliares de enfermería o nutricionistas). Los encuestadores asistieron a una semana de capacitación, en la cual se enseñó el manejo de la herramienta de captura de datos y mantuvieron una comunicación constante con el coordinador del estudio, con un seguimiento semanal acerca de la recolección de la información.

Se obtuvo información sobre la prescripción de medicamentos (denominación, presentación farmacéutica, dosis y periodicidad) en los pacientes atendidos entre el $1 .^{\circ}$ de enero y el 31 de diciembre de 2014. Además, se contó con datos básicos de identificación (edad y sexo) y datos clínicos (tipo de la diabetes, comedicación y su tratamiento).

Los medicamentos fueron categorizados así: (1) biguanidas, (2) sulfonilureas, (3) inhibidores de la dipeptidil peptidasa-4 (iDPP4), (4) agonistas del receptor 
peptido similar al glucagon tipo 1 (AR GLP-1), (5) insulina de acción rápida y (6) insulina de acción prolongada. Los medicamentos prescritos para el tratamiento de las comorbilidades fueron clasificados en (1) antihipertensivos, (2) antiagregantes plaquetarios y (3) hipolipemiantes. Para la categorización de los medicamentos en aquellos grupos se usaron las guías nacionales e internacionales para el tratamiento de la diabetes $[7,8]$.

\section{Análisis de los datos}

El análisis de los datos fue realizado utilizando el programa IBM SPSS $^{\circledR}$ Statistics versión 21 (Armonk, NY: IBM Corp).

Los resultados se presentan en términos descriptivos, mostrando la frecuencia de prescripción de cada una de las categorías de medicamentos.

Para el análisis descriptivo de las variables se determinaron la media, la deviación estándar y las frecuencias.

\section{Consideraciones éticas}

Este protocolo fue sometido y aprobado por el Comité de Ética e Investigaciones de la Pontificia Universidad Javeriana y el Hospital San Ignacio (9 de abril de 2015, Acta 5 de 2015).

De acuerdo con la Resolución 8430 de 1993 del Ministerio de Salud de Colombia, esta investigación fue cataloga como "sin riesgo" [9].

El equipo desarrollador del estudio guardó estricta confidencialidad de la información clínica de los pacientes incluidos, así como de la información de tipo financiero de las instituciones participantes.

Se utilizó una codificación de los pacientes en la base de datos del estudio, que no correspondió a ninguno de los datos de identificación personal de los individuos.

\section{Resultados}

Se revisó la historia clínica de 5361 pacientes con DM, de los cuales 5098 fueron incluidos en este estudio después de aplicar los criterios de exclusión. De este conjunto de pacientes que tuvieron un control médico debido a la DM2, 2859 eran mujeres (56\%), y 2239, hombres (44 \%) (véase tabla 1). La edad media era de 64,9 $\pm 13,1$ años en los hombres y de $64,5 \pm 13,8$ años en mujeres.

La tabla 2 presenta, por sexo de los pacientes con DM2, los medicamentos más utilizados por ellos. Así, los más usados fueron las biguanidas (59\%), las sulfonilureas $(28 \%)$ y las insulinas de acción prolongada (22\%).

Del total de pacientes, $27 \%$ no recibió ningún tratamiento farmacológico para la diabetes, $30 \%$ recibió monoterapia, y $43 \%$, politerapia, en las dos primeras consultas del año 2014 (véase tabla 3). La monoterapia más frecuente fueron las biguanidas $(22 \%$ del total de pacientes en tratamiento y $74 \%$ de aquellos con mono-
Tabla 1. Características de los participantes del estudio.

\begin{tabular}{lccc}
\hline Institución & Hombres & Mujeres & Total \\
\cline { 2 - 4 } $\begin{array}{c}\text { prestadora de } \\
\text { servicios de } \\
\text { salud }\end{array}$ & $(\mathrm{n}=\mathbf{2 2 3 9 )}$ & $(\mathrm{n}=\mathbf{2 8 5 9 )}$ & $(\mathrm{n}=\mathbf{5 0 9 8 )}$ \\
\cline { 2 - 4 } & $\%$ & $\%$ & $\%$ \\
\hline $\mathrm{A}$ & 4,4 & 4,8 & 4,6 \\
$\mathrm{~B}$ & 37,6 & 37,2 & 37,4 \\
$\mathrm{C}$ & 12,3 & 21,6 & 17,5 \\
$\mathrm{D}$ & 43 & 33,8 & 37,8 \\
$\mathrm{E}$ & 2,7 & 2,6 & 2,7 \\
\hline
\end{tabular}

Tabla 2. Patrón farmacológico: uso de medicamentos por pacientes con diabetes mellitus tipo 2 , según sexo, en el año 2014.

\begin{tabular}{lcccc}
\hline & \multicolumn{4}{c}{ Pacientes tratados } \\
\cline { 2 - 5 } & $\mathrm{n}$ & Total & Hombres & Mujeres \\
\cline { 2 - 5 } & 3028 & 59 & 58 & 60 \\
\hline Biguanidas & 1404 & 28 & 28 & 27 \\
Sulfonilureas & 343 & 7 & 6 & 7 \\
iDPP4 & 76 & 2 & 1 & 2 \\
AR GLP-1 & 369 & 7 & 7 & 7 \\
$\begin{array}{l}\text { Insulina de acción } \\
\text { rápida }\end{array}$ & 1105 & 22 & 21 & 22 \\
$\begin{array}{l}\text { Insulina de acción } \\
\text { prolongada }\end{array}$ & 1375 & 27 & 28 & 27 \\
$\begin{array}{l}\text { Ninguna } \\
\text { prescripción }\end{array}$ & & & & \\
\hline
\end{tabular}

iDPP4: Inhibidores de la dipeptidil peptidasa-4; AR GLP-1: Agonistas del receptor del péptido similar al glucagón tipo 1.

terapia). La politerapia más usada fue la combinación de las biguanidas y sulfonilureas, prescrita a uno de cada cinco pacientes con DM2. La segunda opción más reiterada en politerapia fue la combinación de las biguanidas con insulina ( $10 \%$ de los pacientes en tratamiento y $29 \%$ de aquellos con politerapia).

Existe mucha heterogeneidad en los esquemas de tratamiento para la diabetes utilizados en las IPS del país (véase tabla 4). En las IPs B, C y D, la monoterapia más frecuente fueron las biguanidas, y en la IPS A y E, fue iDPP4. En el caso de la politerapia, las combinaciones más usuales para la IPS A fueron las biguanidas y sulfonilureas, las biguanidas e iDPP4; en la IPS B se emplean otras combinaciones: las de las biguanidas con insulinas y sulfonilureas; en la IPS C, al igual que en la IPS D, las combinaciones más comunes fueron las biguanidas y sulfonilureas, o las biguanidas e insulina, y en la IPS E, las combinaciones más recurrentes fueron las biguanidas e iDPP4, o iDPP4 e insulina.

En la tabla 5 se presenta el detalle del patrón de prescripción farmacológica de acuerdo con la medicación para comorbilidades por sexo. Una tercera parte de 
Tabla 3. Patrón farmacológico del paciente con diabetes mellitus en el año 2014, según sexo y terapia.

\begin{tabular}{|c|c|c|c|c|c|}
\hline \multirow{3}{*}{ Terapia } & \multirow{3}{*}{ Medicamentos } & \multicolumn{4}{|c|}{ Pacientes tratados } \\
\hline & & & Total & Hombres & Mujeres \\
\hline & & $\mathrm{n}$ & $\%$ & $\%$ & $\%$ \\
\hline \multirow{6}{*}{$\begin{array}{l}\text { Monoterapia } \\
(\mathrm{n}=1529)\end{array}$} & Biguanidas & 1125 & 22 & 22 & 22 \\
\hline & Sulfinilureas & 161 & 3 & 3 & 3 \\
\hline & iDPP4 & 53 & 1 & 1 & 1 \\
\hline & AR GLP-1 & 1 & 0 & 0 & 0 \\
\hline & Insulina de acción rápida & 10 & 0 & 0 & 0 \\
\hline & $\begin{array}{l}\text { Insulina de acción } \\
\text { prolongada }\end{array}$ & 179 & 4 & 4 & 3 \\
\hline \multirow{10}{*}{$\begin{array}{l}\text { Politerapia } \\
(\mathrm{n}=1801)^{*}\end{array}$} & Biguanidas y sulfonilureas & 1044 & 21 & 22 & 20 \\
\hline & Biguanidas e iDPP4 & 78 & 2 & 1 & 2 \\
\hline & Biguanidas y AR GLP-1 & 12 & 0 & 0 & 0 \\
\hline & Biguanidas e insulina & 527 & 10 & 10 & 11 \\
\hline & $\begin{array}{l}\text { Biguanidas, sulfonilureas } \\
\text { e insulina }\end{array}$ & 73 & 1 & 1 & 2 \\
\hline & Sulfonilureas e insulina & 16 & 0 & 0 & 0 \\
\hline & Sulfonilureas e iDPP4 & 18 & 0 & 1 & 0 \\
\hline & Sulfonilureas y AR GLP-1 & 1 & 0 & 0 & 0 \\
\hline & iDPP4 e insulina & 32 & 1 & 1 & 1 \\
\hline & Otras combinaciones & 393 & 8 & 7 & 8 \\
\hline \multicolumn{2}{|c|}{ Ninguna prescripción $(n=1375)$} & 1375 & 27 & 27 & 27 \\
\hline
\end{tabular}

* Los casos no se presentan de manera excluyente, es decir, un paciente que tenga biguanidas y sulfonilureas, también puede estar en biguanidas e iDPP4.

iDPP4: Inhibidores de la dipeptidil peptidasa-4; AR GLP-1: Agonistas del receptor del péptido similar al glucagón tipo 1.

Tabla 4. Patrón farmacológico del paciente con diabetes mellitus en el año 2014 según institución y terapia.

\begin{tabular}{|c|c|c|c|c|c|c|c|}
\hline \multirow{4}{*}{ Terapia } & \multirow{4}{*}{ Medicamentos } & \multicolumn{6}{|c|}{ Pacientes tratados } \\
\hline & & & \multicolumn{5}{|c|}{ Instituciones prestadoras de servicios } \\
\hline & & & A & $\mathrm{B}$ & C & $\mathrm{D}$ & $\mathrm{E}$ \\
\hline & & $\mathrm{n}$ & $\%$ & $\%$ & $\%$ & $\%$ & $\%$ \\
\hline \multirow{6}{*}{$\begin{array}{l}\text { Monoterapia } \\
(\mathrm{n}=1529)\end{array}$} & Biguanidas & 1125 & 0 & 17 & 15 & 35 & 0 \\
\hline & Sulfinilureas & 161 & 1 & 2 & 6 & 3 & 0 \\
\hline & iDPP4 & 53 & 8 & 0 & 1 & 0 & 45 \\
\hline & AR GLP-1 & 1 & 0 & 0 & 0 & 0 & 0 \\
\hline & Insulina de acción rápida & 10 & 0 & 0 & 0 & 0 & 0 \\
\hline & Insulina de acción prolongada & 179 & 1 & 4 & 4 & 4 & 0 \\
\hline \multirow{5}{*}{$\begin{array}{l}\text { Politerapia } \\
(n=1801)^{\star}\end{array}$} & Biguanidas y sulfonilureas & 1044 & 10 & 9 & 35 & 28 & 0 \\
\hline & Biguanidas e iDPP4 & 78 & 10 & 0 & 0 & 1 & 22 \\
\hline & Biguanidas y AR GLP-1 & 12 & 0 & 0 & 1 & 0 & 0 \\
\hline & Biguanidas e insulina & 527 & 2 & 4 & 24 & 12 & 0 \\
\hline & $\begin{array}{l}\text { Biguanidas, sulfonilureas e } \\
\text { insulina }\end{array}$ & 73 & 2 & 0 & 5 & 1 & 0 \\
\hline
\end{tabular}




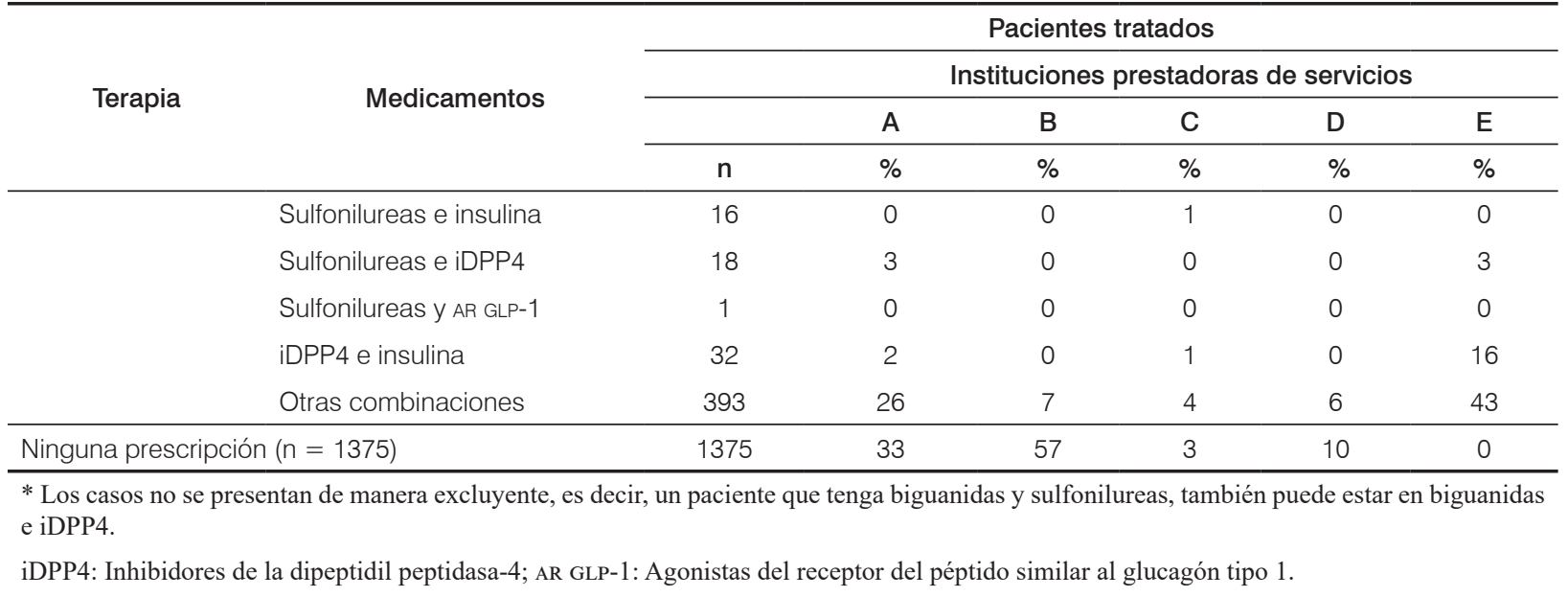

Tabla 5. Patrón farmacológico de los medicamentos para comorbilidades del paciente con diabetes mellitus en el año 2014 según sexo.

\begin{tabular}{lcccc}
\hline & \multicolumn{3}{c}{ Pacientes tratados } \\
\cline { 2 - 5 } Patrón farmacológico de los medicamentos & \multicolumn{2}{c}{ Total } & Hombres & Mujeres \\
\cline { 2 - 5 } & & $\%$ & $\%$ & 54 \\
\hline Uso de medicamentos antihipertensivos & 2713 & 53 & 52 & 38 \\
Uso de medicamentos hipolipemiantes & 2026 & 40 & 42 & 33 \\
Uso de ácido acetilsalicílico & 1828 & 36 & 39 & 10 \\
Uso de medicamento antihipertensivos e hipolipemiantes & 510 & 10 & 10 & 10 \\
Uso de medicamentos antihipertensivos y ácido acetilsalićlico & 525 & 10 & 10 & 17 \\
Uso de medicamentos antihipertensivos, hipolipemiantes y ácido & 961 & 19 & 21 & \\
acetilsalićlico & & &
\end{tabular}

los pacientes con DM2 usa ácido acetilsalicílico, y casi la mitad, antihipertensivos. Además, el $39 \%$ de los hombres y el $33 \%$ de las mujeres con DM2 utiliza al menos un tipo de hipolipemiante. El $19 \%$ de los pacientes tienen los tres tipos de medicamentos (21\% de los hombres y $17 \%$ de las mujeres).

\section{Discusión y conclusiones}

La frecuencia del uso de medicamentos en el tratamiento de la DM en consultas externas de este estudio fue más alta en comparación con las estimaciones de la "Encuesta Nacional de Salud" de 2007, que encontró que a 61,4 \% de los pacientes se le trataba con insulina $u$ otro medicamento [10]. Un análisis de las historias clínicas de los pacientes que asistían a los programas de atención al diabético durante al menos 6 meses, en nueve instituciones de salud entre enero de 2001 y diciembre de 2003 en Medellín (Colombia), reveló que el $15 \%$ de los pacientes estaba sin tratamiento farmacológico [3]. Es muy probable que, dado que los pacientes participaron activamente en un programa, hayan recibido una prescripción.
Los resultados del estudio que aquí se presenta, con respecto a la prevalencia de monoterapia y uso de medicamentos, son diferentes a los reportados en estudios anteriores. El estudio de Villegas et al. encontró que el $44,2 \%$ estaba en esquema de monoterapia, y el $40 \%$ con al menos dos medicamentos [3]. En la formulación de medicamentos se identificó la mayor frecuencia en el uso de las sulfonilureas (58\%), las biguanidas (48\%) e insulina (19,6\%). Las combinaciones más usuales eran de sulfonilureas y biguanidas (en $27 \%$ de los pacientes) y biguanidas con insulina (en $6 \%$ de los pacientes con DM2).

Un estudio observacional del 2005 -de Machado, Moncada y Mesa [4]_, que evaluó la información de más de 7300 pacientes con DM participando en programas de control de la DM, reveló que el 48 \% recibía monoterapia y el $52 \%$ era tratado por DM con asociación de al menos dos medicamentos [4]. Entre los pacientes que recibía monoterapia, la mayor prevalencia de prescripción de medicamentos correspondió a sulfonilureas (42\%), biguanidas $(37 \%)$ e insulina (22\%). En los pacientes que fueron tratados con más de un medicamento antidiabético, las combinaciones más utilizadas incluye- 
ron glibenclamida y biguanidas (75\% de los pacientes), y biguanidas e insulina (13\% de los pacientes). Por frecuencia de prescripción de los medicamentos antidiabéticos, los más formulados fueron biguanidas (en $68 \%$ de los pacientes), glibenclamida (65\%) e insulina (23,5\%).

En este estudio presente, los resultados contrastan con los producidos en un trabajo conducido por Machado, Moncada y Gaviria entre 2006 y 2007, en el cual se analizaron los perfiles de prescripción de medicamentos antidiabéticos para pacientes afiliados al sGSSS, quienes fueron vinculados a programas diferentes de manejo de la DM en nueve ciudades de Colombia [5]. Se encontró, en dicho estudio, que un $46 \%$ de casos fueron tratados con monoterapia. Respecto de la frecuencia de prescripción de los medicamentos antidiabéticos, el uso de las biguanidas fue en el $72 \%$ de los pacientes, mientras el $58 \%$ usaba glibenclamida y $29 \%$ insulina.

A diferencia del estudio de 2005 [4], para la investigación de 2006 y 2007 [5], la fuente de información sobre prescripción de medicamentos antidiabéticos correspondió a la historia clínica de los pacientes.

Lo más probable es que habrá un cambio del patrón farmacológico de los pacientes con DM2 en los próximos años, debido a la disponibilidad de medicamentos nuevos y a la nueva evidencia científica de los últimos años sobre el tratamiento de la DM2, los cuales ya están integrados en la reciente "Guía de práctica clínica para el diagnóstico, tratamiento y seguimiento de diabetes tipo 1, diabetes tipo 2 en mayores de 18 años y diabetes gestacional" [11]. Los resultados de este estudio podrían ser usados como una base para monitorear si los patrones de prescripción farmacológica van a cambiar, implementando las recomendaciones oficiales del tratamiento de la DM2 en Colombia. Esta información podría recolectarse de manera periódica con base en las historias clínicas de las mismas IPS, para investigar si se observan cambios.

Dado que las recomendaciones internacionales señalan empezar a tratar pacientes con DM con biguanidas en combinación con cambios de estilos de vida, la frecuencia del uso de biguanidas en los pacientes de la investigación aquí presentada es muy baja respecto a lo que se muestra en otras investigaciones $[12,13]$. Hay muchas diferencias en los resultados de otros estudios en la región sobre la prescripción de biguanidas o sulfonilureas [14-16]. Por ejemplo: un estudio multicéntrico, transversal, realizado en 3592 pacientes de nueve países de América Latina (Argentina, Brasil, Chile, Costa Rica, Ecuador, Guatemala, México, Perú y Venezuela), en 2006, mostró que los hipoglucemiantes orales utilizados con mayor frecuencia fueron biguanidas (51\%) y sulfonilureas (36\%) [14]. La insulina se utiliza exclusivamente, o en combinación con un agente oral, en un 14,5\% de los pacientes.
Asimismo, un análisis de datos de 13513 pacientes con DM de centros de atención primaria públicos y privados de la seguridad social de Argentina, Brasil, Chile, Colombia, Paraguay y Uruguay, en el año 2001, mostró que, entre los pacientes tratados con medicamentos antidiabéticos, los fármacos orales de monoterapia más usados fueron las sulfonilureas (33\%), las biguanidas (9\%) y su administración combinada (14\%) [15].

Un estudio descriptivo de 332 pacientes con diagnóstico de DM2 con tratamiento farmacológico en México, en el 2005, reveló que las sulfonilureas fueron el hipoglucemiante oral más prescrito [16]. Este mismo estudio, además, reportó que solo el $54 \%$ de los pacientes siguió la prescripción correcta de hipoglicemiantes orales según los criterios de la norma oficial mexicana.

Hay suficientes datos científicos para concluir que el ácido acetilsalicílico es eficaz en la disminución de la morbilidad y la mortalidad cardiovascular en pacientes que están clasificados de alto riesgo, con infarto de miocardio previo o aquellos con accidente cerebrovascular [13]. Sin embargo, su beneficio en la prevención primaria en pacientes sin eventos cardiovasculares previos es más discutido, tanto para los que tienen diabetes como los sin diabetes $[17,18]$.

Las recientes directrices de tratamiento publicadas por la Asociación Americana de la Diabetes aconsejan usar ácido acetilsalicílico en hombres y mujeres mayores de 50 años con diabetes tipo 1 o tipo 2, y uno o más factores de riesgo importantes (antecedentes familiares de enfermedad cardiovascular, hipertensión arterial, tabaquismo, dislipidemia o albuminuria), y que no tienen un mayor riesgo de sangrado [13,19-22]. Sin embargo, no se recomienda el ácido acetilsalicílico para aquellos con bajo riesgo de enfermedad cardiovascular, dado que los beneficios probablemente son superados por un riesgo de sangrado [13].

A pesar de las recomendaciones basadas en resultados obtenidos en estudios científicos, el $35 \%$ de las pacientes con DM tuvieron una prescripción de aspirina en este estudio. Dado que la mayoría de los pacientes con DM de este estudio tuvo edad superior a 50 años y por eso ya posiblemente se presentan con al menos uno de los factores de riesgo de las enfermedades cardiovasculares, hay que investigar la razón de la baja prescripción de la aspirina. Estudios previos en pacientes con DM en Colombia mostraron una prevalencia de la prescripción del ácido acetilsalicílico entre 2,8 y $66,1 \%$ [4,5].

Una limitación del presente estudio es que hubo historias clínicas con información incompleta, sin datos clínicos ni de medicamentos. Además, dado que algunos pacientes no cumplieron con todas las citas anuales de control, no fue posible obtener toda la información necesaria sobre las prescripciones. Tampoco se evaluó la adherencia a la medicación o efectos secundarios, dado 
el tipo de estudio. No se consiguió información sobre la duración de la DM2 o control de la DM2. Por eso, las diferencias en el uso de los medicamentos entre las IPS pueden eventualmente ser debidas al control de la DM2, o a comorbilidades, o todas las anteriores. Sin embargo, el objetivo de este trabajo fue presentar un perfil general del patrón farmacológico en los IPS participantes.

En conclusión, los resultados de este estudio podrían servir al desarrollo de estrategias para el tratamiento y el seguimiento adecuado del paciente con DM.

\section{Agradecimiento}

Agradecemos a las siguientes instituciones, por permitirnos tener acceso a sus registros médicos: Fundación Cardioinfantil-Instituto de Cardiología, Bogotá, Colombia; Fundación Oftalmológica de Santander, Bucaramanga, Colombia; Hospital Universitario San Ignacio, Pontificia Universidad Javeriana; Centro de Diabetología, Barranquilla; Centro Integral de Diabetes, Medellín, Colombia; Funcentra, Montería, Colombia, y Centro Médico Oftalmológico, Ibagué, Colombia.

\section{Referencias}

1 International Federation of Diabetes. IDF diabetes atlas. 7. $^{\mathrm{a}}$ ed. Bruselas: IDF; 2015. p. 15.

2 Aschner P. Epidemiología de la diabetes en Colombia. Av. Diabetol. 2010;26(2):95-100.

3 Villegas-Perrasse A, Abad S, Faciolince S, Hernández N, Maya C, Parra L, et al. El control de la diabetes mellitus y sus complicaciones en Medellín, Colombia, 2001-2003. Rev. Panam. Salud Pública. 2006;20(6):393-402.

4 Machado-Alba J, Moncada-Escobar J, Mesa-Escobar G. Patrones de prescripción de antidiabéticos en un grupo de pacientes colombianos. Rev. Panam. Salud Pública. 2007;22(2):124-31.

5 Machado-Alba J, Moncada-Escobar J, Gaviria H. Quality and effectiveness of diabetes care for a group of patients in Colombia. Rev. Panam. Salud Pública. 2009;26(6):529-35.

6 Mejía S, Vélez A, Buriticá O, Arango M, Río J del. La política farmacéutica nacional en Colombia y la reforma de la seguridad social: acceso y uso racional de medicamentos. Cad. Saúde Pública 2002;18(4):1025-39.

7 American Diabetes Association. Pharmacologic approaches to glycemic treatment. Diabetes Care 2017;40(Suppl. 1):S64-S74.

8 Aschner P, Muñoz V, Girón D, García O, Fernández-Ávila D, Casas $\mathrm{L}$, et al. Guía de práctica clínica para la prevención, diagnóstico, tratamiento y seguimiento de la diabetes mellitus tipo 2 en la población mayor de 18 años. Colombia Médica 2016;47(2):109-31.

9 Colombia, Ministerio de Salud y Protección Social. Resolución 8430 de 1993. Por la cual se establecen las normas científicas, técnicas y administrativas para la investigación en salud. [Internet] 1993 [citado 2014 ago. 10]. Disponible en: https://www.minsalud.
gov.co/sites/rid/Lists/BibliotecaDigital/RIDE/DE/DIJ/RESOLUCION-8430-DE-1993.PDF

10 Colombia, Ministerio de la Protección Social. Encuesta Nacional de Salud (ENS) 2007. [Internet] 2009 [citado 2014 ago. 10]. Disponible en: https://www.minsalud.gov.co/Documentos $\% 20 \mathrm{y} \% 20$ Publicaciones/ENCUESTA\%20NACIONAL.pdf

11 Colombia, Ministerio de Salud y Proteccion Social. Guía de práctica clínica para el diagnóstico, tratamiento y seguimiento de la diabetes mellitus tipo 2 en la poblacion mayor de 18 años. Bogota: Alianza CINETS. [Internet] 2015. [citado 2014 ago. 10]. Disponible en: http:// gpc.minsalud.gov.co/gpc_sites/Repositorio/Conv_637/GPC_diabetes/DIABETES_TIPO_2_COMPLETA.pdf

12 Guzmán JR, Lyra R, Aguilar-Salinas CA, Cavalcanti S, Escaño F, Tambasia M, et al. Treatment of type 2 diabetes in Latin America: A consensus statement by the medical associations of 17 Latin American countries. Rev. Panam. Salud Pública. 2010;28(6):463-71.

13 American Diabetes Association. Standards of medical care in diabetes 2016. Diabetes Care 2016;39(Suppl. 1): S1-112.

14 Lopez-Stewart G, Tambascia M, Rosas Guzmán J, Etchegoyen F, Ortega-Carrión J, Artemenko S. Control of type 2 diabetes mellitus among general practitioners in nine countries of Latin America. Rev. Panam. Salud Pública. 2007;22(1):12-20.

15 Gagliardino JJ, Hera M de la, Siri F y Grupo de Investigación de la Red QuAlidiab. Evaluación de la calidad de la asistencia al paciente diabético en América Latina. Rev. Panam. Salud Pública 2001;10(5):309-17.

16 Gómez-García A, Soto-Paniagua JC, Álvarez-Aguilar C. Uso de hipoglucemiantes orales en pacientes con diabetes mellitus tipo 2 . Aten. Primaria 2005;35(7):348-52.

17 Antithrombotic Trialists' (АтT) Collaboration, Baigent C, Blackwell L, Collins R, Emberson J, Godwin J, et al. Aspirin in the primary and secondary prevention of vascular disease: Collaborative meta-analysis of individual participant data from randomised trials. Lancet 2009;373(9678):1849-60.

18 Perk J, De Backer G, Gohlke H, Graham I, Reiner Z, Verschuren $\mathrm{M}$, et al. European Guidelines on cardiovascular disease prevention in clinical practice (version 2012). The Fifth Joint Task Force of the European Society of Cardiology and other societies on cardiovascular disease prevention in clinical practice (constituted by representatives of nine societies and by invited experts). Eur. Heart J. 2012;33(13):1635-701.

19 Huxley RR, Peters SA, Mishra GD, Woodward M. Risk of allcause mortality and vascular events in women versus men with type 1 diabetes: A systematic review and meta-analysis. Lancet Diabetes Endocrinol. 2015;3(3):198-206.

20 Peters SA, Huxley RR, Woodward M. Diabetes as risk factor for incident coronary heart disease in women compared with men: A systematic review and meta-analysis of 64 cohorts including 858,507 individuals and 28,203 coronary events. Diabetologia. 2014;57(8):1542-51.

21 Kalyani RR, Lazo M, Ouyang P, Turkbey E, Chevalier K, Brancati $\mathrm{F}$, et al. Sex differences in diabetes and risk of incident coronary artery disease in healthy young and middle-aged adults. Diabetes Care. 2014;37(3):830-8.

22 Peters SA, Huxley RR, Woodward M. Diabetes as a risk factor for stroke in women compared with men: A systematic review and meta-analysis of 64 cohorts, including 775,385 individuals and 12,539 strokes. Lancet 2014;383(9933):1973-80. 